\title{
Vom Sinn und Unsinn eines flächendeckenden Tiers payant in der Schweiz
}

Urs Stoffel

Co-Präsident der Konferenz der Kantonalen ÄrzteGesellschaften $\mathrm{KKA} / \mathrm{CCM}$

\section{Einleitung}

Grundsätzlich gibt es in der Schweiz im ambulanten Bereich zwei Abrechnungssysteme der Kostenvergütung im Obligatorischen Krankenpflegebereich (OKP). Diese beiden Vergütungsarten sind im KVG im Art. 42 geregelt. Das System, bei dem der Versicherte dem Leistungserbringer die Vergütung der Leistung schuldet, wird Tiers garant (TG) genannt. Die Versicherten haben in diesem Fall einen Anspruch auf eine Rückerstattung durch die Versicherung. Wenn keine anderen Vereinbarungen zwischen dem Leistungserbringer und dem Kostenträger vereinbart wurden, so gilt gemäss KVG dieses System als Regelfall.

Gemäss Absatz 2 Art. 42 KVG können aber Versicherer und Leistungserbringer vereinbaren, dass der Versicherer die Vergütung schuldet. Dieses System heisst Tiers payant (TP).

Mehrheitlich wird in der Schweiz nach dem System des TG abgerechnet. In den letzten Jahren kommt nun zunehmend die Forderung der Versicherer nach dem Abrechnungssystem des TP. Von der Seite der Versicherungen wird argumentiert, dass in diesem System, insbesondere durch die Einführung des elektronischen Datenaustausches (eDA) über einen Intermediär (MediData), prämienwirksam Kosten gespart werden können.

\section{Es ist volkswirtschaftlicher Unsinn, wenn alle Arzt- rechnungen direkt zu den Krankenversicherungen gehen}

Korrespondenz: Dr. med. Urs Stoffel Seestrasse 49 CH-8002 Zürich Tel. 0442862020 urs.stoffel@hin.ch
Seit geraumer Zeit bestehen erhebliche Differenzen zwischen den Versicherern und der Ärzteschaft über die Abwicklung des eDA im TG via die ärzteeigenen TrustCenter und im von den Versicherern geforderten TP über den Intermediär MediData, der von den Versicherern betrieben und finanziert wird. Auf diese Thematik werde ich an dieser Stelle nur kurz und vor allem zur Frage des Datenschutzes eingehen. Zahlreiche Stellungnahmen und Gegendarstellungen zur Problematik TrustCenter versus MediData wurden bereits öffentlich thematisiert [1]. In diesem Artikel möchte ich aber aus ärztlicher Sicht der Frage nachgehen, wie sinnvoll die Einführung eines flächendeckenden Tiers payant im schweizerischen Gesundheitswesen wirklich ist.

\section{Volkswirtschaftlicher Unsinn}

Aus Sicht der Ärzteschaft ist es ein volkswirtschaftlicher Unsinn, wenn alle Arztrechnungen automatisch und direkt $\mathrm{zu}$ den Krankenversicherungen gehen (System des TP). Wie wir aus verschiedenen Untersuchungen wissen, ist der Anteil von kleinen Rechnungen (unter 100 Franken) in Arztpraxen im OKPBereich sehr hoch und der TP führt deshalb nur zu teuren administrativen Umtrieben ohne jeglichen Nutzen. Im Gegensatz zum UVG-Bereich, der keinen Selbstbehalt kennt, müssen die Rechnungen im KVGBereich zuerst mit der Franchise und dem Selbstbehalt verrechnet werden.

Ein grosser Anteil der im TP direkt an die Versicherung geschickten Rechnungen muss mit einer komplizierten Abrechnung dem Patienten erneut in Rechnung gestellt werden, weil ein grosser Teil der Arztrechnungen unterhalb der Franchisekosten pro Jahr liegt. Das ist Bürokratie pur und bläht lediglich den Verwaltungsapparat der Krankenversicherungen künstlich auf. Und die prämienwirksamen Kosten steigen, ohne dass irgendein gesundheitlicher Nutzen für die Versicherten generiert würde. Allerdings steigt der Umsatz der Krankenversicherungen. Und das hat aus Sicht der Versicherer den positiven Effekt, dass der prozentuale Anteil der Verwaltungskosten im Verhältnis zum Umsatz sinkt!

\section{Stärkung der Eigenverantwortung und Entlastung der Prämien}

Auch in den jüngsten Diskussionen des Parlaments für kostendämmende Massnahmen im Gesundheitswesen und vor allem im Zusammenhang mit der Schaffung von differenzierten Selbstbehalten wird immer wieder betont, wie wichtig die Stärkung der Eigenverantwortung für das Kostenbewusstsein der Bevölkerung sei. Es wird gefordert, dass kleine Beiträge aus der eigenen Tasche («out of the pocket») bezahlt werden sollen, so dass die Gesundheitskosten und damit die Prämien nicht unnötig belastet werden. Ein flächendeckender TP widerspricht diesen Forderungen nach Eigenverantwortung. Denn jeder Franken, der über den Betrag der Franchise hinausgeht, wird in diesem System von den Versicherungen bezahlt und wirkt sich damit direkt auf die Prämien aus. Untersuchungen mit santésuisse haben gezeigt, dass eine Differenz von bis zu 15\% zwischen den gestellten Rechnungen und den bei den Versicherungen zur Rückvergütung eingereichten Rechnungen besteht. Es ist also davon auszugehen, dass bei einem 
flächendeckenden System des TP die prämienwirksamen Kosten und im speziellen die Verwaltungskosten deutlich steigen werden.

\section{Ein flächendeckender Tiers payant widerspricht den Forderungen nach Eigenverantwortung}

\section{Kostenkontrolle und Kostenbewusstsein}

Niemand kann eine Arztrechnung besser kontrollieren als die Patientin, an der die Leistung erbracht wurde. Die neuste Umfrage von santésuisse hat entgegen den Erwartungen ergeben, dass 70\% der Patienten ihre Arztrechnungen kontrollieren. Solange die Patientin primäre Schuldnerin bleibt (TG) wird sie diese Rechnung als erste erhalten und diese (wie alle Rechnungen bei denen sie direkte Schuldnerin ist) prüfen. Wenn aber der Patient im System des TP irgendwann, wenn überhaupt, meist nachdem die Kasse längst bezahlt hat, eine Kopie der Rechnung oder die Leistungsabrechnung des Versicherers erhält, interessiert er sich nicht mehr dafür. Die Kasse hat ja längst bezahlt. Er ist sich damit auch nicht mehr wirklich bewusst, welche Kosten er ausgelöst und verursacht hat. Auch der gewünschte Anreiz zur Kosten- und Qualitätskontrolle und zum erhöhten Kostenbewusstsein geht im System des TP verloren.

\section{Datenschutz und Schutz des Bürgers vor dem «gläsernen Patienten»}

Durch den äusserst filigranen und differenzierten TARMED-Tarif ist der Patient heute zu weiten Teilen zum «gläsernen Patienten» geworden. Aus den detaillierten Rechnungspositionen lässt sich oft die Diagnose und damit auch die Erkrankung und sogar die Prognose der Patientin ableiten. Nach wie vor gibt es Behandlungen und Leistungen (z.B. Psychiatrie, Aids-Test, Bagatellbehandlungen usw.) von denen der Patient nicht möchte, dass die Versicherung davon Kenntnis hat (z.B. auch im Zusammenhang mit Zusatzversicherungen) und die der Patient aus der eigenen Tasche bezahlen will. Nachdem auch vom Datenschützer eine Freigabe jeder einzelnen Rechnung respektive Behandlung gefordert wird, sind die heute häufig gebrauchten generellen Entbindungen vom Datenschutz gegenüber dem Arzt (Pauschal- ermächtigung) nicht zulässig. In einem flächendeckenden TP ist die Ermächtigung der Patientin pro Rechnung nicht mehr möglich und deshalb haben wir im TP ganz generell ein klares Datenschutzproblem. Dieses Datenschutzproblem wurde mehrfach vom eidgenössischen Datenschützer in seinen Berichten moniert und er empfiehlt die Abwicklung der Arztrechnungen im TG über ein TrustCenter. An dem erst kürzlich erfolgten Treffen mit dem eidgenössischen Datenschützer hat Hanspeter Thür diese Forderungen erneut und dezidiert bestätigt. Aus seiner Sicht können die Datenschutzauflagen im TP nur erfüllt werden, wenn der Patient vor der Übermittlung der elektronischen Rechnung an den Versicherer eine Rechnungskopie erhalten hat.

\section{Wir haben im Tiers payant generell ein klares Datenschutzproblem}

\section{Elektronischer Datenaustausch}

Seit 2004 stellt die frei praktizierende Ärzteschaft ihre Rechnungen datenschutzkonform und in elektronischer Form zum Abholen in den von den Ärzten selbstfinanzierten TrustCentern bereit. Diese Form des elektronischen Datenaustausches wurde auch ausdrücklich vom eidgenössischen Datenschützer in seinem Bericht vom Juni 2004 gutgeheissen und sogar empfohlen. Mehrere Versicherer nutzen diesen Weg des eDA seit Jahren rege, mit gutem Erfolg und zu ihrer vollsten Zufriedenheit. Im Mai 2010 ist die 100-millionste Rechnung in den TrustCentern eingetroffen. Es lagern damit über eine Milliarde Datensätze in den ärzteeigenen TrustCentern. Jährlich gehen bei den TrustCentern 19 Millionen Rechnungen ein. Damit ist und bleibt das grösste Sparpotential im eDA die Nutzung der in den TrustCentern datenschutzkonform bereitgestellten elektronischen Rechnungen durch die Versicherungen.

\section{Literatur:}

1 Stoffel U. Was ist uns die Datenparität wert? Schweiz Ärztezeitung. 2003;84(28):1510-1.

2 Helfer G. Tiers payant versus Tiers garant - Fragwürdiges Verhalten der Ärztegesellschaften. PrimaryCare. 2005;5(43):890-1. 